\title{
THE FLOCCULATION OF TURBID LIQUIDS BY SALTS.
}

\author{
BY A. D. HALL, M.A. AND C. G. T. MORISON, B.A.
}

The Rothamsted Experimental Station.

IT has long been recognised that a trace of soluble salt will bring about the flocculation of the material suspended in a turbid liquid; and because of its bearing upon such practical matters as the clearing of drinking water, the deposition of silt at the mouths of estuaries, and the improvement of the texture of heavy soils, the process has received a considerable amount of investigation. It is probably related to the flocculation of colloids by similar reagents; the fine particles of clay which chiefly cause the turbidity of natural waters being composed of a hydrated silicate of alumina and other bases possessing properties akin to those of the colloids. Indeed Schloesing has proposed to call that portion of clay material which remains obstinately suspended in water, sometimes for days together, 'colloid clay,' as something distinct in kind from the other particles in the soil. However it is reasonable to suppose that the distinction between the 'colloid' clay and the rest is in the main one of size, the colloid particles being so small as to be beyond the limits of microscopic vision, just as the true colloids may be regarded as consisting of very large molecules, lying between the molecules which go into solution and the molecular aggregates that remain in water as suspended solids.

Various theories have been proposed to account for the phenomenon of flocculation (see Schulze, Journ. Prac. Chem. 25 (1882), 431; Picton and Linder, Trans. Chem. Soc. 67, 1895, p. 63; Whetham, "Theory of Solutions," Phil. Mag. v. 48, 474 (1899); Joly, Compt. Rend. du VIII Congrès Geologique International, 1900; Spring, Rec. Trav. Chim. Pays Bas, 1900 , p. 222, which paper also contains a bibliography), but since they did not seem to accord with other observations on the behaviour 
of saline solutions towards clay, the authors decided to re-examine the whole subject.

Since the flocculation of 'natant' material and the clearing of the turbid liquid is a question of degree, depending on such factors as the strength of the flocculating salt solution, time, temperature, \&c., no absolute measure of flocculation can be obtained. Only a comparative method of experiment can be adopted, and much of the difficulty of correlating the work already done lies in the want of definition of what the author has meant by flocculation. For the present investigations a standard material was first prepared: this consisted of a purified kaolin from which the coarser particles had been separated by decantation in water; it would remain suspended in a column of water $7.5 \mathrm{~cm}$. high for more than 24 hours and was made up of particles less than $0.002 \mathrm{~mm}$. in diameter.

A stock of this having been prepared and suspended in a large bulk of water, 10 c.c. were withdrawn for each experiment after brisk shaking, a quantity that was found to contain 0.2219 gram. of kaolin.

The experiments were carried out in glass cylinders holding 200 c.c. of water to which the 10 c.c. of kaolin suspension were added together with a measured quantity of a standardised solution of the flocculating salt, the whole being then thorougbly stirred. The cylinders stood in an unheated room free from vibration or much change of temperature, and after a few hours, when clearing had sufficiently progressed, each jar was matched by eye with one or other member of a standard series. This standard series was made up each time of a number of cylinders in which the flocculation was effected by regular increments of calcium nitrate, so as to obtain concentrations lying between $n / 40,000$ and $n / 2500$. Thus by preliminary trials and by varying the concentration of the substance under examination it could be matched against one of the standard jars and could be brought in to a comparative numerical scale indicating its flocculating power, on the assumption that this flocculating power is inversely proportional to the amount of substance required to produce the standard effect. Thus if a concentration $=n / 2000$ normal of a substance $A$ is required to produce the same result as a concentration of $n / 10,000$ calcium nitrate, it is assumed that the flocculating power of $\boldsymbol{A}$ is only one-fifth that of calcium nitrate. The photographs, Plate IV, Fig. 1, show the character of the results obtained in three series of these experiments. It was found to be important to work with equal quantities of standard material of fairly uniform fineness of grain; the relations are obscured if the amount of 
material varies too widely, or if it contains many coarser particles, because of their tendency to drag finer ones down with them on settlement.

Only certain substances seem to be capable of indefinite suspension in water, of assuming what the authors call the 'natant' condition, however finely divided they may be. For example among the class of bodies likely to be found in soils, hydrated ferric oxide, obtained by grinding natural limonite or iron rust into a very fine state, flocculated spontaneously and settled down rapidly after shaking up with water. Yet the fineness of grain was such as would allow it to assume the natant condition, and indeed it did become so if a little ammonia were added to the water. Hydrated alumina, in the form of finely ground bauxite, behaved in a similar manner, and silica after long rubbing in an agate mortar did not yield wholly satisfactory results; even the finest precipitated silica makes a suspension that remains turbid for but a short time. In a truly 'natant' suspension the particles are just defined when enlarged one thousand diameters; they are then seen to be in rapid Brownian motion, but if a trace of flocculating salt be introduced at the edge of the cover-glass as it diffuses into the field of view the particles will be observed to lose their motion and draw together into little clots. These aggregates are however not permanent, but can be at once broken up so as to restore the 'natant' condition by washing away the flocculating salt.

This reversibility distinguishes true flocculation from cases of the clearing of an opalescent liquid by the growth of the suspended particles, such as is seen when a dilute silver solution is precipitated as chloride and the presence of an excess of acid induces the accretion of the very fine particles.

The following experiments illustrate the nature of the changes taking place during flocculation.

1. Up to a certain point, flocculation, as measured by the rapidity of settlement, is proportional to the amount of Hocculating salt added. Beyond that limit all solutions flocculate alike.

Suspensions were made up in the usual way by mixing 200 c.c. of water with 10 c.c. of the strong kaolin suspension and a measured quantity of calcium nitrate solution to bring the strength of the liquid to the concentration specified in the second column. 
JOURNAL OF AGRICULTURAL SCIENGE. Vol. II. No. 3.

PLATE IV
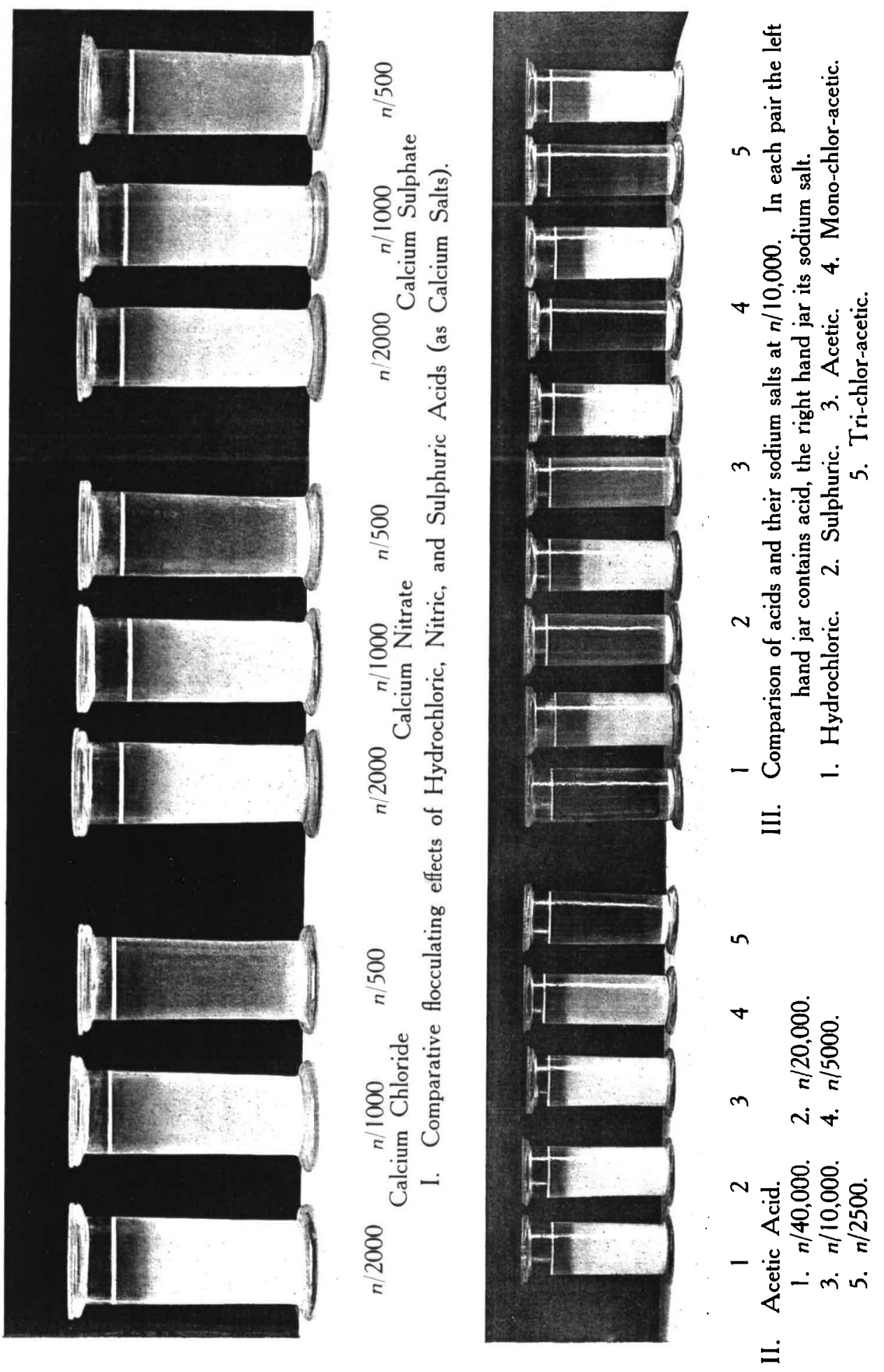
Experiment I. Calcium nitrate.

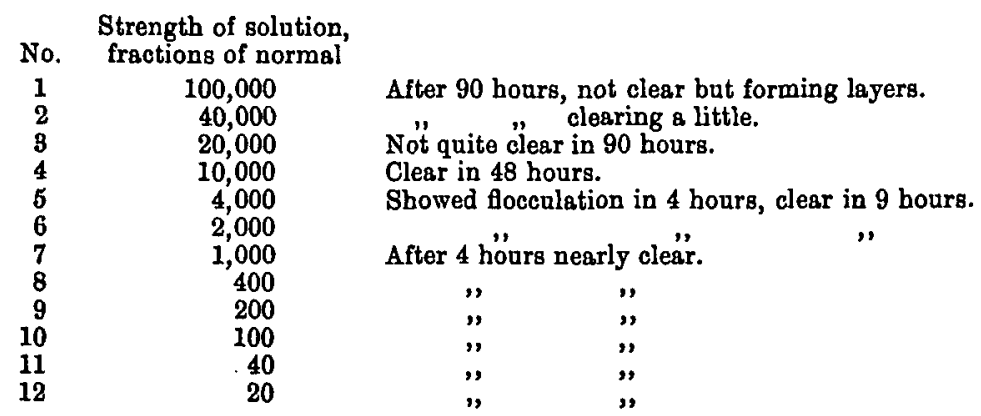

In this case a concentration of $n / 1000$ seems to be about the limit for the amount of kaolin used, increase of concentration beyond that point does not cause any quicker settlement. This experiment may be compared with a parallel series where sodium chloride replaced the calcium nitrate.

\section{EXPERIMENT II. Sodium chloride.}

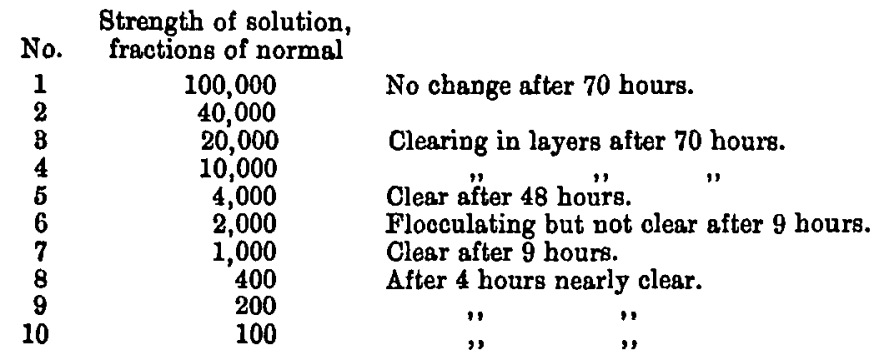

Here the limit may be taken as $n / 400$ sodium chloride, as compared with $n / 1000$ calcium nitrate, all the suspensions containing the two salts in these ratios pursuing a parallel course.

The results with calcium nitrate and sodium chloride were typical of those obtained with a number of other salts, the only variation being the limit of concentration above which complete flocculation set in. Of course some salts will not induce flocculation at all, a fact which will be considered later.

No evidence was obtained of reversal effects, such as a substance ceasing to flocculate when its concentration was increased above a certain strength, or of the existence of successive maximal and minimal points of flocculation as described by Joly (loc. cit.), though experiments were made with sodium nitrate up to concentrations of $2 n$ or 17 per cent. of the salt. 
2. The reaction between the flocculated substance and the flocculant is a quantitative one.

The following experiment will illustrate this point; the amount of kaolin in the suspensions was varied, being 1, 2, 4, and 8 times the normal respectively. Similarly the amount of the flocculating salt, calcium nitrate, was varied so as to obtain three series of jars containing the same ratio of kaolin to salt, the water being varied to make up the same bulk in each case.

\section{EXPERIMENT III.}

$\begin{array}{cccc}\text { No. } & \begin{array}{c}\text { c.c. of kaolin } \\ \text { suspension }\end{array} & \begin{array}{c}\text { c.c. of } n / 100 \text { calcium } \\ \text { nitrate added }\end{array} & \begin{array}{c}\text { Ratio of kaolin to } \\ \text { calcium nitrate }\end{array} \\ 1 & 1 & 1 & 1 \\ 2 & 2 & 1 & 2 \\ 3 & 4 & 1 & 4 \\ 4 & 8 & 1 & 8 \\ 5 & 2 & 2 & 1 \\ 6 & 4 & 2 & 2 \\ 7 & 8 & 2 & 4 \\ 8 & 4 & 4 & 1 \\ 9 & 8 & 4 & 2 \\ 10 & 8 & 8 & 1\end{array}$

Under these conditions $1,2,3,4$ cleared in succession though they all contained the same concentration of calcium nitrate. Numbers 1,5 , 8 and 10, however, cleared approximately together, as afterwards did 2,6 , and 9 , and still later 3 and 7 . Suspensions containing the same ratio of kaolin to calcium nitrate cleared simultaneously, whatever the absolute amounts of the substances that were present in the liquid. In other words, a certain amount of salt is required to flocculate a given amount of kaolin, a conclusion which would also follow from the existence of a limit of concentration required to bring about flocculation in a given kaolin suspension, as described in the previous section. To carry out this experiment successfully it is of course necessary not to overpass this limit, otherwise all the suspensions will settle at the same rate. Again it is necessary to work at great dilutions and with small quantities of kaolin, because the relationship becomes obscured by the tendency of the higher concentrations of kaolin to clear more rapidly. When the flocculated kaolin is in any quantity it mats together, falls quickly, and to some extent clears the liquid behind it. In other words, a dense turbid liquid clears more effectually than a thin one, and this is true whether it is flocculated or not. But with care the quantitative relationship can be verified for various salts.

3. The process of flocculation does not appear to be accompanied by any removal of the salt from solution by the flocculated kaolin 
(adsorption or laking out), nor by any selective absorption of base from the salt, so as to give rise to acidity in the liquid after flocculation had taken place. Picton and Linder (loc. cit.) found that the flocculation of arsenious sulphide by barium chloride was accompanied by a withdrawal of barium from solution, so that the resulting medium was acid. Working with kaolin no such acidity could be detected, and other experiments in the Rothamsted Laboratory (see Hall and Gimingham, Trans. Chem. Soc., 91, 1907, 677) show that soluble salts such as cause flocculation merely interchange bases with the complex hydrated double silicates which make up kaolin.

Experiments were also made to ascertain if a change in conductivity accompanied the process of flocculation, as follows:-

A cylinder of Jena glass holding about 300 c.c. was immersed in a constant temperature bath with glass sides. A pair of platinum electrodes, held rigid by tubes (of Jena glass) containing the leads and suitable cross-pieces above and below, were completely immersed in the cylinder, which also contained a stirrer of silver. Usually the experiment began by measuring the conductivity of a measured volume of pure water in the cylinder, and determining the increase brought about by the addition of 10 c.c. of a solution of flocculating salt kept in the same bath. The required amount of kaolin suspension, also kept in the same bath, was then run in and the change in conductivity determined again. A previous blank experiment gave the conductivity of the kaolin suspension when diffused alone in the given volume of pure water.

Two of the experiments may be given, conductivities being expressed in reciprocal ohms per c.c.

\section{EXPERIMENT IV. Barium chloride (n/350) and kaolin (0.2 gram).}

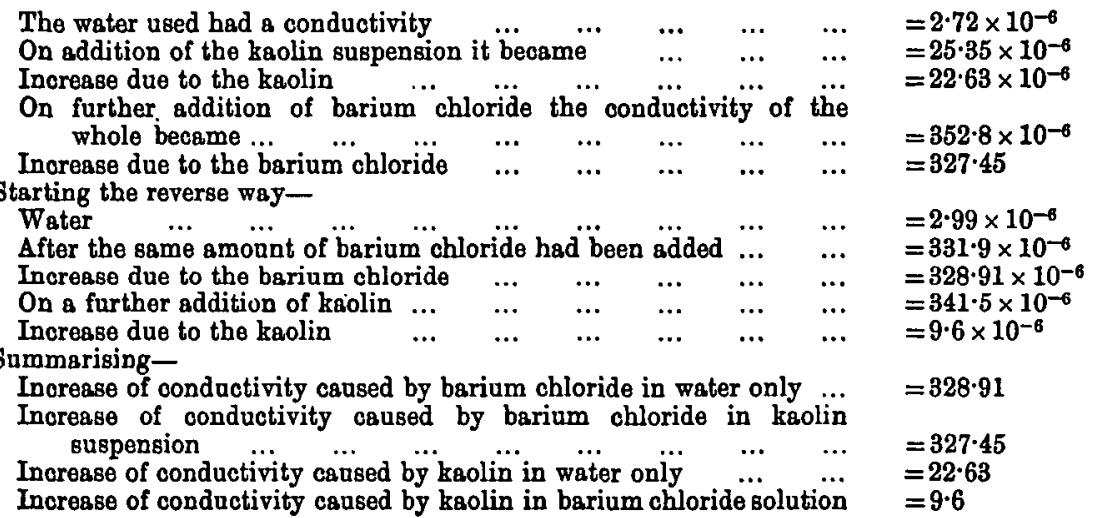


Thus in neither case did the flocculation result in lowering the conductivity of the salt solution, though when the kaolin was added to the barium chloride the increase in conductivity was not quite so great as would be expected from simply adding the conductivities of the separate constituents together.

EXPERIMENT V. Aluminium sulphate (n/350) and kaolin $(0.2$ gram).

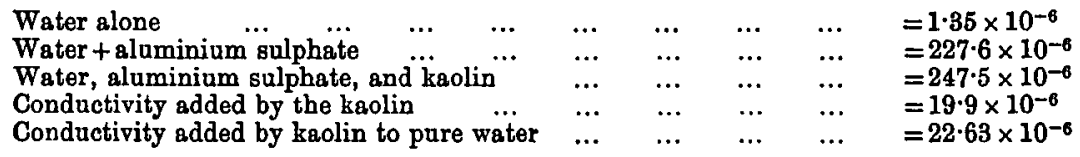

ExpERIMENT VI. Finely powdered natrolite $(0 \cdot 2$ gram $)$ and water.

$\begin{array}{llllllllll}\text { Water alone } & \ldots & \ldots & \ldots & \ldots & \ldots & \ldots & \ldots & \ldots & =2.72 \times 10^{-6}\end{array}$

$\begin{array}{lllllllll}\text { Water and natrolite } & \ldots & \ldots & \ldots & \ldots & \ldots & \ldots & \ldots & =2.62 \times 10^{-6}\end{array}$

Increase of conductivity due to natrolite alone $\begin{array}{lllll}\ldots & \ldots & \ldots & \ldots & =681 \times 10^{-8}\end{array}$

EXPERIMENT VII. Natrolite $(0.2$ gram) and barium chloride $(n / 350)$.

$\begin{array}{llllllll}\text { Barium chloride solution } & \ldots & \ldots & \ldots & \ldots & \ldots & \ldots & =396.0 \times 10^{-6}\end{array}$

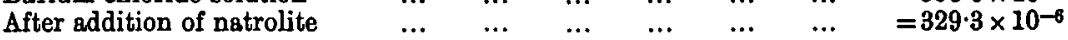

EXPERIMENT VIII. Natrolite $(0.2 \mathrm{gram})$ and ammonium chloride $(n / 350)$.

$\begin{array}{llllllll}\text { Ammonium chloride solution } & \ldots & \ldots & \ldots & \ldots & \ldots & \ldots & =390.8 \times 10^{-6}\end{array}$

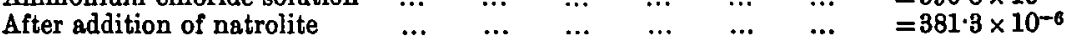

In the two latter cases the addition and flocculation of natrolite were accompanied by a slight reduction in the conductivity of the solution, but this may be explained by the fact that barium or ammonium in the solution was to some extent replaced by sodium from the natrolite, i.e. by an element with a lower specific conductivity at those dilutions employed.

Other experiments were tried, but there was no evidence of any such drop in conductivity as would be occasioned by the removal from solution of any appreciable fraction of the salt.

4. The next stage in the experiments was to ascertain the comparative flocculating powers of a series of salts. The method of work was in all cases the same; varying amounts of the salt under investigation were added to the jars containing suspensions of kaolin made in the usual way, and these were matched against a standard series started at the same time with varying amounts of calcium nitrate as flocculant. It was early found that the flocculating power of the salts of a given metal is not independent of the acid, so that in 
the comparisons of the various metals, chlorides had to be tested against chlorides, sulphates against sulphates, and so on.

The following list shows the comparative flocculating power of equi-normal solutions of the various metals tried, when the effect of the acid is eliminated; calcium nitrate being taken as a standard $=10$.

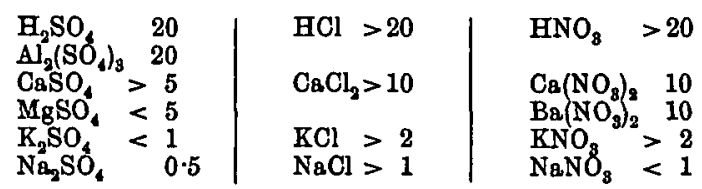

The most powerful flocculators are therefore the free acids, though the aluminium salts come very close, possibly because they are so completely hydrolised in solution. The trivalent aluminium is more effective than the divalent elements calcium and magnesium, which in their turn flocculate better than sodium or potassium; but there is no evidence for the ratios $1: 32$ :: 1024 between mono-, di- and trivalent elements, which were found by Picton and Linder and justified on theoretical grounds by Whetham. Moreover the monovalent elements do not agree among themselves, potassium being about twice as effective as sodium, and hydrogen ten times greater still.

It should be remembered that the dilution of the solutions causing flocculation in these experiments is very great, usually about onethousandth normal, so that the salts must be regarded as completely ionised.

It has already been stated that the flocculating power of a salt depends on the nature of the acid as well as of the base; a number of experiments were made to ascertain the relative order of the three acids -hydrochloric, nitric and sulphuric. The same relation was obtained whether the free acids or their salts were used, the best measurements of the ratios being

$\begin{array}{ll}\text { Hydrochloric acid } & 20 \\ \text { Nitric acid } & 19 \\ \text { Sulphuric acid } & 13\end{array}$

These ratios are not unlike the relative ionic dissociations of the three acids, as measured by their electrical conductivities, but any theory founded on such an agreement is not borne out by the behaviour of other acids like acetic. Acetic acid proved to be a very effective flocculant, being but little less active than sulphuric acid, 9 as against 13 on the above scale. Substitution of chlorine in the acetic acid made but 
little difference, monochloracetic acid being rather more effective than the di- and tri-chloro acids, and all being a little better than acetic acid itself. The range of difference can hardly however be expressed in figures. On the other hand, amid-acetic acid did not flocculate at all.

Of the other acids tried oxalic and tartaric caused flocculation, and may be represented by 2.5 on the scale, oxalic being rather the more effective. Citric acid and phenol did not flocculate at all.

Perhaps the most interesting case is however afforded by the behaviour of the hydrates: it has always been known that the free alkaline hydrates are powerful deflocculators, a trace of ammonia, potash or soda will immediately cause clay particles to assume the 'natant' condition. This is also true of the carbonates and bi-carbonates of the alkalis and of similar salts of weak acids like the borates and the phosphates. Calcium hydrate is however used in practice to flocculate clay, as is shown by the improved tilth resulting from the application of quicklime to clay soils. In this case however the action is complicated by the presence of carbon dioxide, which will on the one hand precipitate the calcium as carbonate, dragging down at the same time the suspended material, and again will give rise to soluble calcium bi-carbonate.

Several determinations were made of the flocculating power of calcium hydrate, care being taken to deprive all the solutions used as far as possible of carbon dioxide and also to keep the suspensions out of contact of the air during their standing. Under these conditions calcium hydrate had a flocculating power of about 3 , when calcium nitrate is rated at $\mathbf{1 0}$ for comparison; the concentration of the suspension in calcium hydrate being about one-thousandth normal. It will thus be seen that the flocculating effect of calcium hydrate is positive, whereas the hydrates of potassium, sodium, and ammonium give negative effects. Barium hydrate gave a very similar result to calcium hydrate.

It may perhaps be concluded that hydroxyl ions have a negative flocculating action, which is able to overpower the positive action of the potassium, sodium and ammonium ions, but is not equal to the greater positive action of the calcium or barium ions.

When calcium bi-carbonate was tried it proved a very effective flocculator, being a little better than equivalent solutions of calcium nitrate, though a numerical value could not be given to the superiority. Nor could this be attributed to the excess of carbon dioxide, for 
measurements of a solution of free carbon dioxide only give the low value of 0.5 for carbon dioxide.

5. A few experiments were tried with other materials than kaolin. Bauxite and limonite respectively were ground fine in an agate mortar, and further reduced by wet grinding in an improvised ball-mill for a day or two. Precipitated silica was also prepared and used after igniting and grinding. Neither bauxite nor limonite would assume the 'natant' condition in pure water; they flocculated and settled immediately. The presence of alkali in concentrations of $n / 2000$ to $n / 500$ however caused them to remain suspended; sulphuric acid flocculated, but aluminium sulphate at the same concentrations caused the bauxite to remain suspended. In a second series the bauxite with aluminium sulphate again held up better than the check with pure water, but magnesium sulphate tried at the same time permitted of flocculation like the pure water. A small sample of allophane, $\mathrm{Al}_{2}(\mathrm{HO})_{2} \mathrm{SiO}_{4}+x \mathrm{H}_{2} \mathrm{O}$, was scraped off and reduced to a fine powder; this also would not assume the natant condition in pure water, though it wonld remain suspended when a trace of free alkali was added.

The experiments with these bodies were abandoned because of the impossibility of obtaining true suspensions in water alone.

With ignited silica there was no difference between the suspensions in pure water and those containing either acid or alkali; flocculation could be brought about by aluminium sulphate, but it was difficult to make satisfactory suspensions.

The authors have failed to find any satisfactory theory that embraces the whole of the observed facts.

The electrical theory developed by Whetham, depending upon the discharge of the suspended particles by the free ions, would seem to fail, because

(1) The flocculation depends upon the ratio of amounts of flocculating material and flocculant, and not merely on the concentration of the flocculant.

(2) The different acids and the salts of different acids show great variations in their flocculating power, nor are these variations dependent on the degree to which ionisation takes place. Acetic acid and the acetates for example have much the same value as sulphuric acid and the sulphates, and chlorinating the acetic acid has little effect on the result.

(3) The ratio between the flocculating power of mono-, di- and trivalent metals is far from that expected by the theory. 
(4) No change takes place in the conductivity of the solution during and after flocculation.

The electrical theory sketched by Joly is similarly not justified by the facts.

Again flocculation cannot depend upon the hydrolysis of the salts employed and the action of the free acid, except perhaps in the case of the aluminium salts. Hydrolysis alone would not explain the effectiveness of such salts as calcium chloride which are but slightly hydrolised, nor the high position of the acetates, nor the fact that the chloracetates possess much the same vulue as the acetates.

The authors also consider that the conductivity experiments and the quantitative measurements with stronger solutions already referred to must dismiss any theory founded on the selective precipitation of the base of the flocculant on to the suspended particles and a resulting 'laking out.'

Nor can any theory be built up on an hypothesis of preliminary chemical action between the salt and the particles (though the salts in question do act upon kaolin), accompanied by aggregation. Such an explanation is sufficiently negatived by the fact that the action is reversible; the flocculated material can be suspended afresh in pure water when the salt has been washed away.

It was thought that the preliminary chemical action might induce some change in the surface tension existing between the solid particles and the solution; this however is not a sufficient hypothesis as the following experiment shows. Kaolin was flocculated by $1 / 10,000$ normal ammonium chloride solution, washed free from salt, and resuspended in pure water. On adding ammonium chloride to again bring up the solution to $1 / 10,000$ normal the kaolin was flocculated afresh. No new chemical action could have taken place, because on the first occasion the kaolin would have completed its reaction with the ammonium chloride of that concentration so that no further change would take place on a second contact with another solution of the same strength.

Before any theory of flocculation can be reached it is probably necessary to determine the conditions which must be satisfied before a given substance will assume the 'natant' state. While size is of course a factor, for particles above about $0.004 \mathrm{~mm}$. will not remain suspended for any length of time, chemical composition also comes in to determine whether the particles below this limit will possess a Browuian motion and remain apart instead of aggregating when 
suspended in water. Of the substances examined, only kaolin natrolite and the various clays, which may be regarded as impure kaolins, would become natant; hydrated alumina, hydrated ferric oxide, silica, and even allophane, another hydrated silicate of alumina, fall rapidly and completely from suspension to form a matted deposit at the bottom of the vessel. Furthermore it can be shown that it is not the zeolitic character of the kaolin \&c. that confers the natant character. A sample of kaolin was ignited for some time at a low red heat, more than sufficient to destroy any zeolitic compounds; this sample was then graded by suspension in water like the original kaolin and a fraction separated that would remain suspended for 24 hours. A number of suspensions were then made up containing equal dilutions of ignited and unignited kaolin respectively, and various amounts of calcium nitrate added to induce flocculation; the ignited kaolin assumed a true 'natant' condition, but was more readily flocculated than the unignited kaolin. The only feature that seems to distinguish the natant from the non-natant bodies, is that the former-kaolin and natrolite-are essentially double salts of an alkaline metal which give rise to a trace of free soluble alkali when they are mixed with water. Both kaolin and natrolite contain alkaline metals, and fresh mineral specimens when ground in an agate mortar with pure water will gradually blue a strip of red litmus paper immersed in the thin paste when it is allowed to dry spontaneously. On the other hand the specimen of allophane used seemed to be free from the alkali metals, as also are limonite, bauxite and the silica used; these latter substances would only become natant when free alkaline hydrates were added to the suspensions.

If then the natant condition is dependent on the presence of traces of free alkali derived from the partial hydrolysis of the suspended material, it is clear that the free acids should be the best flocculators; it is also reasonable to suppose that the presence of a salt, which would react in the way of double decomposition with the part of the material that would be hydrolised by pure water, would also tend to suppress any free alkaline hydrates. Again the general quantitative relationship between suspended material and flocculant would also follow; even the departure from this rule in that a concentrated suspension requires a smaller proportion of flocculant than a dilute one, can be explained by assuming that the amount of hydrolysis of the natant material would be greater when it was in contact with a larger bulk of water. 
Bejond this point, that the natant condition is dependent upon the presence of free alkaline hydrates and that flocculation ensues when these are neutralised or driven back iu to combination with the suspended solid, the authors prefer to attempt to put forward no further theory, but merely to record a series of observations and measurements that are critical of the theories already advanced, in the hope that they may afford material to induce some physicist to take up the question afresh. It would appear to be necessary first to arrive at an explanation of Brownian motion. 